\title{
RIS SYNERGY: STANDARDS SCHAFFEN - SERVICES DIGITALISIEREN - EXPERTISE VERNETZEN
}

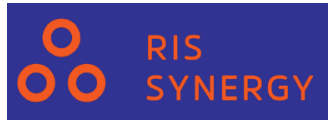

\section{von Madeleine Harbich, Ulrike Hicker und Sabine Neff}

Zusammenfassung: Das Projekt „RIS Synergy"verfolgt zwei wesentliche Aspekte digitaler Transformation: Im Teilprojekt „Schnittstellen und Standards" werden offene Zugangs- bzw. Austauschmöglichkeiten für Systeme von Fördergebern, Forschungsstätten und der öffentlichen Verwaltung geschaffen. Das Teilprojekt „Konzeptstudie Forschungsportal" befasst sich mit Rahmenbedingungen und Anforderungen eines international sichtbaren und vernetzten Forschungsportals mit dem Ziel, In- und Output der Forschungslandschaft darzustellen und innovative Vernetzungsmöglichkeiten zu schaffen.

Schlagworte: Digitalisierung; Transparenz; Standardisierung; Schnittstellen; Forschung; RIS; Forschunsinformationssystem; Once-Only; CERIF; Forschungsportal; Datenaustausch; Publikationsdaten

\section{RIS SYNERGY: CREATING STANDARDS - DIGITIZING SERVICES - NETWORKING EXPERTISE}

Abstract: "RIS Synergy" deals with two essential aspects of digital transformation: The sub-project "Interfaces and Standards" creates open access and exchange options for systems of funding agencies, research institutions, and public administration. The second sub-project "Concept study research portal" designs the framework conditions and requirements of an internationally compatible research portal. It aims at the input and output of the Austrian research landscape and wants to create innovative networking opportunities.

Keywords: Digitalization; Transparency; Standardization; Research; RIS; Research Information System; Once-Only; CERIF; Research Portal; Data Exchange; Publication Data

DOI: https://doi.org/10.31263/voebm.v74i2.6269

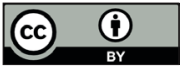

Dieses Werk ist - exkl. einzelner Logos und Abbildungen - lizenziert unter einer Creative-Commons-Lizenz Namensnennung 4.0 International-Lizenz 


\section{Einleitung}

Fragen Sie sich oft, was mit den Daten, die Sie mühsam an so vielen Stellen eingeben, passiert? Warum muss alles mehrfach erfasst werden, wenn doch alle Informationen bereits digital vorliegen? Die Antwort darauf ist: der Schein trügt leider, denn selbst wenn wir längst genug Wissen darüber und die Fähigkeiten dazu haben, die meisten Systeme tauschen leider noch keine Informationen aus.

Stellen Sie sich vor, wir Universitäten könnten Publikationsmetadaten weitergeben, ohne dafür einen Finger rühren zu müssen. Unsere Forscher*innen könnten Publikationsmetadaten in unserem CRIS (Current Research Information System, englisch für Forschungsinformationssystem) erfassen und gleichzeitig ihre Berichtspflicht bei der Forschungsfördergeber*in erfüllen ohne Daten mehrfach eintragen zu müssen. Stellen Sie sich vor, dass mit dieser einfachen Eingabe auch ihr Profil in einem zukünftigen nationalen Forschungsportal ergänzt wird. Der Benefit liegt auf der Hand: mühsame Mehrfacherfassung und der damit verbundene Datenverlust können vermieden und die Datenqualität durch bidirektionalen Datenabgleich deutlich gesteigert werden. Informationen, die nicht an der Universität erhoben werden (können), finden zurück in das System und ergänzen das Gesamtbild. Standardisierung ermöglicht eine Vielzahl an weiteren Anwendungen in der Zukunft. Diese Vision ist ein Teil unserer Arbeit im Digitalisierungsprojekt RIS Synergy.

\section{Nachhaltiger Umgang mit Forschungsdaten \& -informationen}

Forschung produziert und verarbeitet Daten. Während sich Daten über Forschende und ihre Aktivitäten in den universitären Forschungsinformationssystemen finden, werden die im Rahmen der Forschung gewonnenen Daten und Erkenntnisse in Repositorien verwaltet. Ein Zusammenspiel beider Systeme, ein strukturiertes Forschungsdatenmanagement sowie der Einsatz von zukunftsweisender Infrastruktur und Services ist Voraussetzung für den nachhaltigen Umgang mit Forschungsdaten.

Aus diesem Grund denken Österreichs Universitäten das Thema größer: RIS Synergy ist Teil eines Projektclusters (Cluster Forschungsdaten ${ }^{1}$ ), der sich gesamtheitlich mit dem Umgang mit Forschungsdaten und -informationen auseinandersetzt. 


\section{Entlastung und Sichtbarkeit für den Wissenschaftsbetrieb}

Das Projekt RIS Synergy schafft Entlastung und Sichtbarkeit für den Wissenschaftsbetrieb durch das Festlegen von Standards, die Digitalisierung von Services und die Vernetzung von Expertise.

An dem im März 2020 an der TU Wien gestarteten Projekt sind bundesweit 11 Universitäten und drei Fördergeber beteiligt. Durch standardisierten Austausch von Metadaten werden die Forschenden entlastet, die Effektivität und Qualität im Forschungssupport erhöht und die Datenqualität zu Forschungsoutput verbessert. RIS Synergy schafft dadurch die nachhaltige und zukunftsweisende Basis, für die Digitalisierung der österreichischen Forschungslandschaft und ermöglicht die internationale Sichtbarmachung österreichischer Forschungserfolge.

\subsection{Keine Mehrfacherfassung - Once-Only!}

Im Teilprojekt „Schnittstellen und Standards“ werden offene Zugangs- bzw. Austauschmöglichkeiten für Systeme von Fördergebern, Forschungsstätten und der öffentlichen Verwaltung geschaffen. Im Fokus stehen die Datenerfassung nach dem Once-Only-Prinzip sowie der Austausch von Informationen zu Förderprogrammen, Organisationsstrukturen, Daten aus e-Call-Systemen, Datenmanagementplänen und Metadaten zu Forschungsoutputs.

Teil hiervon sind neben administrativen Informationen und Projektdaten auch die bereits erwähnten Publikationsmetadaten. Auf Grund der Vielzahl unterschiedlicher Formen von Forschungsoutput (Publikationen, Forschungsdaten, Vorträge, Patente, ...) beginnt das Projekt zunächst mit der Umsetzung einer Schnittstelle für Publikationen mit DOI (Digital Object Identifier; zahlenmäßige Codierung). Eine Vorbedingung für die Übermittlung über die Schnittstelle zwischen Universität und Fördergeber* in ist die Erfassung und Validierung des DOIs von Forschungsoutput und dessen Relation zum Projekt im CRIS der Forschungsstätten.

Damit Forscher*innen zukünftig ihre Berichtspflichten sowohl innerhalb der Forschungseinrichtung als auch für die Drittmittelförderung ohne Mehrfacherfassung (wie bisher üblich) erfüllen können, wird an einer Schnittstelle gearbeitet, die dem europäischen Standard CERIF (Common European Research Information Format) entspricht. Zukünftig soll ein*e Forscher* in den DOI einer im CRIS erfassten Publikation mit gemeinsamen Projekt IDs verknüpfen. Diese Daten werden der*dem Fördergeber* in über die Schnittstelle bereitgestellt. Der hiermit geschaffene Mehrwert kann zukünftig als Modell für weiteren Forschungsoutput und Schnittstellen zu 
anderen Systemen übernommen werden. Durch die Verknüpfung von Programm- und Projektinformationen mit Forschungsoutput wird eine durchgängige Information in Sinne des Projektlebenszyklus ermöglicht.

Der Once-Only-Faktor könnte sich, neben vielen weiteren Verwendungen, auch bis in die Vision für ein nationales Forschungsportal weiterziehen. Hierfür erarbeitet RIS Synergy bis Februar 2024 ein Konzept, das die Bedürfnisse und Anforderungen der österreichischen Forschungslandschaft kritisch hinterfragt und zukunftsweisende Lösungen anbietet. Im Fokus der Arbeit steht auch hier der Wunsch des Projektkonsortiums Mehrfachaufwand zu reduzieren, Synergien zu nutzen, Netzwerke aufzubauen und den Wissenschaftsstandort Österreich zu stärken.

\section{Die Konzeptstudie in RIS Synergy}

Die Ergebnisse des Teilprojekts „Schnittstellen“ stellen bereits einen essentiellen Mehrwert im Zusammenhang mit digitalen Forschungsförderungsprozessen dar, legen aber auch die Basis für ein Forschungsportal, über das sich In- und Output der Forschungslandschaft digitalisiert und transparent darstellen lassen und welches an internationale Standards anschlussfähig ist. Daher wird im 2. Teilprojekt eine Konzeptstudie durchgeführt, in der Rahmenbedingungen, Anforderungen und Funktionalitäten eines österreichweiten Forschungsportals definiert werden.

\subsection{Potentiale eines nationalen Forschungsportals}

Nationale Portale ermöglichen in einigen Ländern bereits Vernetzung und transparente Bereitstellung von Information. ${ }^{2}$ In Österreich muss jede Universität selbst ihre Forschungsleistung und die dahinterstehenden Forschenden sichtbar machen. Die Idee, ein nationales Forschungsportal schrittweise aufzubauen, würde eine neutrale und transparente Darstellung des In- und Outputs von Forschungsprojekten ermöglichen. Nationale Forschungserfolge könnten zentral kommuniziert und somit in ihrer Gesamtheit sichtbar gemacht werden. Von einer gemeinsamen Sichtbarkeit würden auch kooperative Initiativen und Netzwerke, z.B. FIS/CRIS Austria - Netzwerk der österreichischen FIS/CRIS-Systeme, OANA - Open Science Network Austria (bald: OSA - Open Science Austria) etc., und weitere Open Science-Initiativen profitieren. Die Forschungsstätten erhielten die Chance, über das Portal transparente, differenzierte und qualitative Dokumentation und Präsentation von Forschungsleistung gegenüber der 
öffentlichen Verwaltung und Politik zu bieten und damit ihre Außenwahrnehmung selbst mitzugestalten.

Doch so ein nationales Forschungsportal könnte noch mehr leisten. Als Vernetzungsplattform zur strategischen Bündelung von Forschungskompetenz, zur Förderung nachhaltiger Kooperationsgemeinschaften und als Kommunikationsplattform für gemeinsame Aktivitäten (z.B. „Intention of Submission", Initiativen in Pre-Call-Phasen, etc.) könnte man mit einem nationalen Portal Synergien schaffen, die eine einzelne Institution schwer bieten kann und auch jungen Forscher*innen neue Chancen öffnen würde.

Ein nationales Forschungsportal würde außerdem ein gesundes Gegengewicht zur zunehmenden Dominanz kommerzieller Anbieter bei Forschungsinformationssystemen schaffen. Zudem wäre da noch der Austausch der Daten, der RIS Synergy auch im Teilprojekt 1 beschäftigt und hier bereits als Basis vieler Nutzungsmöglichkeiten dienen könnte. Ein nationales Forschungsportal könnte Schnittstelle zur nationalen Forschungsinfrastrukturdatenbank, Open Data-Netzwerken, etc. sein und für internationalen Datenaustausch genutzt werden.

Letztlich könnte der Einstieg über ein gemeinsames Portal der Ausgangspunkt eines übersichtlichen und einfachen Zugangs für Forschende zu allgemeinen Informationen und Services sein - im Sinne eines „OneStop-Shop"-Prinzips - und somit eine große Lücke im Forschungsalltag schließen.

Das Teilprojekt 2 „Konzeptstudie Forschungsportal“ wurde im März 2021 gestartet. Bisher wurden drei Themengruppen gebildet, die in einem ersten Schritt zur Erarbeitung eines Konzeptes für ein nationales Forschungsportal dreierlei Aspekte beleuchten:

In der Arbeitsgruppe „Zielgruppenanalyse und Umfrage“ widmen sich die Mitarbeiter*innen den möglichen Zielgruppen eines nationalen Forschungsportals mittels einer systematischen Stakeholderanalyse. Dabei werden auch der Bedarf und die Möglichkeiten für diese Zielgruppen beleuchtet. In weiterer Folge soll eine Umfrage bei den identifizierten Zielgruppen durchgeführt werden, um die Annahmen zu verifizieren und die genauen Bedarfe und Anforderungen zu ermitteln.

Dem Vergleich anderer nationaler Forschungsportale widmet sich eine zweite Arbeitsgruppe. Ziel ist es hier aus den Erfahrungen der Anderen zu lernen. Ausgewählte, bereits etablierte nationale Forschungsportale in anderen Ländern werden genauer beleuchtet, um Erkenntnisse für Österreich abzuleiten und Best Practices herauszuarbeiten.

In einer dritten Arbeitsgruppe, „Lessons learned, Kooperationen und Synergien“, werden vergangene und ergänzende Initiativen zu einem nati- 
onalen Forschungsportal in Österreich beleuchtet, und die Kooperation mit anderen laufenden (Digitalisierungs-)Projekten gesucht, um Synergien zu nutzen und Redundanzen zu vermeiden. Bisher wurde Kontakt mit dem Projekt $\mathrm{AT} 2 \mathrm{OA} 2^{3}$ aufgenommen, wobei bereits erste wesentliche Aspekte zur Zusammenarbeit identifiziert wurden.

\section{Projektstand und Ausblick}

Das Projektkonsortium hat bis dato alle Meilensteine erreicht und die als umfangreiche Basis geplante Analysephase abgeschlossen. Erste Umsetzungen werden vorangetrieben und der Kreis der Projektpartner wird laufend erweitert. Interessent*innen stehen alle Bereiche des Projekts zur Mitarbeit offen, und Input aus weiteren Blickwinkeln ist ausdrücklich erwünscht, um bestmögliche Ergebnisse sicherzustellen. Mit der Pilotumsetzung einer Finanzdatenschnittstelle werden bereits 2021 Entlastungen im Zusammenhang mit der Projektadministration erzielt. Bis 2024 werden noch weitere Erleichterungen durch Schnittstellen in weiteren Bereichen des Forschungszyklus folgen. Mit dem Startschuss für das Teilprojekt 2 im März 2021, befindet sich nun auch die Konzeptstudie für ein nationales Forschungsportal in der Analysephase. Projektübergreifend ist der Benefit des großen Netzwerks, das RIS Synergy umfasst, spürbar und sichert nachhaltige Umsetzungen. Alle Planungen erfolgen mit Fokus auf nationale Zusammenarbeit und internationale Standards.

Madeleine Harbich, Bakk ${ }^{\mathrm{a}}$

Universität Wien, Forschungsservice und Nachwuchsförderung E-Mail: madeleine.harbich@univie.ac.at

Mag. ${ }^{a}$ Ulrike Hicker

TU Wien, Forschungsinformationssysteme E-Mail: ulrike.hicker@tuwien.ac.at

Dipl.-Ing. ${ }^{\text {in Sabine Neff }}$

TU Wien, Forschungsinformationssysteme E-Mail: sabine.neff@tuwien.ac.at

Mitteilungen der VÖB 74 (2021) Nr. 2: Digitale und soziale Transformation 


\section{Projektname: RIS Synergy}

Projektlaufzeit: 2020-2024

Projektpartner: 11 Universitäten und 3 Fördergeber (FWF - Der Wissenschaftsfonds, FFG - Die Österreichische Forschungsförderungsgesellschaft, WWTF - Wiener Wissenschafts- und Technologiefonds, TU Wien, Universität Wien, Universität Klagenfurt, Universität Innsbruck, Universität Salzburg, Medizinische Universität Graz, Veterinärmedizinische Universität Wien, Universität für Musik und darstellende Kunst Wien, Akademie der bildenden Künste Wien, Universität für angewandte Kunst Wien, TU Graz

\section{Ziele:}

- Digitalisierung und Steigerung der Effektivität bei der Betreuung von Forschungsprojekten

- Ressourcenschonung für die Wissenschaft und Steigerung der Wettbewerbsfähigkeit der Forschenden

- Steigerung der Datenqualität und Transparenz für Forschungsstätten, Fördergeber und öffentliche Stellen

- Standardisierte, offene Schnittstellen und Umsetzung des OnceOnly-Prinzips

- Nachhaltiger Umgang mit Daten im Sinne der DSGVO

- Darstellung von In- und Output der Forschungslandschaft nach internationalen Standards (CERIF)

- Erarbeitung einer gemeinsamen Position hinsichtlich eines nationalen Forschungsportals

\section{Benefits aus dem Projekt:}

- Entlastung der Forschenden und des administrativen Universitätspersonals durch standardisierten Austausch von Metadaten

- Erhöhung der Effektivität und Qualität im Forschungssupport

- Verbesserung der Datenqualität zu Forschungsoutput

- Sichtbarmachung nationaler Erfolge und Forschungskompetenz

1 Cluster Forschungsdaten: https://forschungsdaten.at/

2 euroCRIS, Directory of Research Information System (DRIS): https:// dspacecris.eurocris.org/browse?type $=$ drisscope

3 AT2OA2: https://www.at2oa.at/at2oa2_home.html 